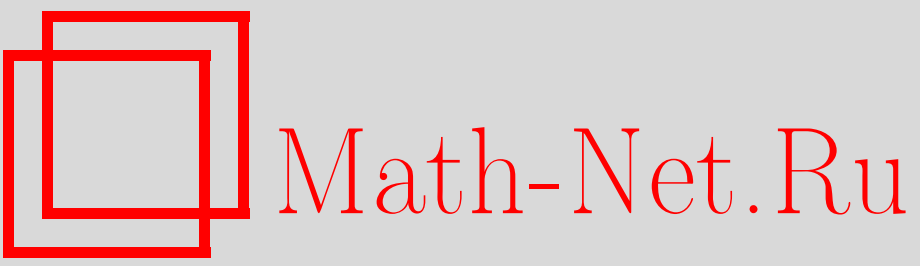

С. В. Найденов, В. В. Яновский, Геометро-динамический подход к бильярдным системам. I. Проективная инволюция бильярда. Прямая и обратная задачи, TMФ, 2001, том 127, номер 1, 110-124

DOI: https://doi.org/10.4213/tmf451

Использование Общероссийского математического портала Math-Net.Ru подразумевает, что вы прочитали и согласны с пользовательским соглашением

http://www.mathnet.ru/rus/agreement

Параметры загрузки:

IP : 54.92 .164 .108

26 апреля 2023 г., 13:04:31 
ТЕОРЕТИЧЕСКАЯ

И МАТЕМАТИЧЕСКАЯ

ФИЗИКА

Том 127, № 1

апрель, 2001

(c) 2001 г. $\quad$ С.В. Найденов*, В.В. Яновский*

\title{
ГЕОМЕТРО-ДИНАМИЧЕСКИЙ ПОДХОД К БИЛЬЯРДНЫМ СИСТЕМАМ. І. ПРОЕКТИВНАЯ ИНВОЛЮЦИЯ БИЛЬЯРДА. ПРЯМАЯ И ОБРАТНАЯ ЗАДАЧИ
}

\begin{abstract}
В рамках предложенного геометро-динамического подхода к бильярдам как к обратимым динамическим системам специального вида установлена их связь с проективными преобразованиями (инволюциями). Сформулированы прямая и обратная задачи для бильярдов. Получены уравнения, определяющие решения этих задач в общем виде. Вычислены некоторые простейшие бильярдные инволюции. Установлены функциональные зависимости между инволюцией бильярда, уравнением его границы и полем нормалей к ней. Выявлена связь инволюции с кривизной границы бильярда.
\end{abstract}

\section{1. ВВЕДЕНИЕ}

Бильярд как динамическая система - это популярная и глубокая модель для физических систем различной природы от газа сталкиваюшихся шаров (в статистической механике) или совокупности нуклонов (в короткодействующем потенциале атомного ядра) до резонаторов и волноводов, в том числе квантовых, для частиц и излучений и т.п. Большое значение бильярд имеет, с одной стороны, как классическая модель [1]-[4] хаотической динамики, а с другой - как перспективная система, на которой отрабатываются возможные сценарии перехода к квантовому (в квазиклассическом приближении) (см., например, [5]) и многомерному хаосу [6], [7].

По сушеству, бильярд является геометро-динамической системой. Он определяется как формой границы, так и простейшим законом прямолинейного движения с упругими (зеркальными) отражениями от его гранищы. Очевидно, геометрические закономерности такой системы тесно переплетаются с ее динамическими свойствами. Особую роль играет связь бильярда с проективными преобразованиями, к которым относятся обычные евклидовы движения и гомотетия. Проективные преобразования непосредственно связаны с упругим характером отражения для бильярдного движения.

${ }^{*}$ Институт монокристаллов НАН Украины, Харьков, Украина 
Наиболее естественный подход при исследовании бильярда - это сведение его к специальной динамической системе (точечному отображению или каскаду). Возникающие при этом отображения являются сохраняющими меру отображениями. В предыдущих исследованиях бильярдов [1]-[4], [8]-[10] указанные системы предполагались консервативными (якобиан отображения считался строго равным единище). В предложенном и используемом здесь подходе бильярд отождествляется с проективной инволюцией - обратимым отображением на специально введенном фазовом пространстве с симметричными (равноправными) координатами. Это пространство является многообразием $M$, представленным в виде прямого произведения $M=\partial \Omega \otimes \partial \Omega$, топология которого зависит от геометрии границы бильярда $\partial \Omega$. Любая бильярдная траектория определяется как последовательность итераций этого бильярдного отображения, примененных к произвольно выбранной начальной точке. Последняя принадлежит введенному фазовому пространству, а ее первая и вторая координаты на многообразии $M$ определяют соответственно точки падения и последующего отражения луча геометрической траектории от границы бильярда, параметризованной каким-нибудь удобным способом в указанных координатах. Среди преимушеств предложенного подхода можно отметить следующие. Отображение, вызванное дискретностью бильярдных отражений, получается непосредственно без промежуточных переходов вначале к гамильтонову потоку в кокасательном расслоении бильярда, а затем к его трансверсальным сечениям. Отображение возникает как естественный переход к сокрашенному описанию системы, так как движение между отражениями не рассматривается. Однако потери полноты динамической информации при этом непроисходит, и реальные траектории всегда можно восстановить целиком по парам точек отражения, соответствующих точкам фазового пространства, причем даже в случае непрямолинейного характера распространения луча между отражениями (например, “магнитные" бильярды, бильярды во внешнем поле, таком как гравитационное, или бильярды на кривых поверхностях). Обе координаты фазового пространства, являясь, по существу, конфигурационными переменными, задающими положение точек отражения на границе бильярда, оказываются в известном смысле более равноправными по отношению друг к другу. Это роднит их с лагранжевыми координатами, в отличие от канонически-сопряженных переменных гамильтонова подхода. Аналогичной особенностью обладает и само полученное ниже бильярдное отображение, с ней же связан более удобный вид его симметричного (относительно диагонали отображения) фазового портрета. Наконец, в этом подходе выявляются наиболее существенные черты произвольного бильярда - проективность и инволютивность (обратимость), что позволяет выделить и исследовать бильярдные системы в отдельном классе отображений специального вида.

Геометрическое движение по бильярдной траектории обратимо во времени. Обратимость бильярда неразрывно связана с его проективными свойствами (об обратимых системах и их важной роли в физике см., например, [11]-[13]). В частности, в подходящих координатах определяющая бильярд инволюция сводится к дробно-рациональной зависимости, а соответствие между падающим и отраженным лучами является гармоническим проективным преобразованием. 
Бильярд полностью и однозначно определяется заданием его инволюции (это будет доказано ниже). Для плоской односвязной замкнутой границы $\partial \Omega \in \mathbb{R}^{2}, \partial \Omega \sim S^{1}$ с классом гладкости $\mathcal{C}^{r+1} \quad(r \geqslant 0)$ соответствуюшее бильярду отображение с такой инволюцией имеет вид отображения на торе $T^{2}$ :

$$
\begin{gathered}
B:=\left\{\begin{array}{l}
\bar{\varphi}_{1}=\varphi_{2} \quad(\bmod 2 \pi), \\
\bar{\varphi}_{2}=f\left(\varphi_{1}, \varphi_{2}\right) \quad(\bmod 2 \pi),
\end{array}\right. \\
\left(\varphi_{1}, \varphi_{2}\right) \in T^{2}=S^{1} \times S^{1}, \quad \vec{r}=\vec{r}(\varphi) \in \partial \Omega \sim S^{1}, \\
f\left[f\left(\varphi_{1}, \varphi_{2}\right), \varphi_{2}\right]=\varphi_{1} \Leftrightarrow f \circ f=\mathrm{id}, \quad f \in \mathcal{C}^{r}\left(T^{2} ; S^{1}\right),
\end{gathered}
$$

где $\vec{r}(\varphi)$ - одна из допустимых параметризаций границы бильярда $\partial \Omega$ с ориентированной кривизной $\widehat{K}, f\left(\varphi_{1}, \varphi_{2}\right)$ - инволюция бильярда. Для бильярда с произвольной непрерывной границей $\partial \Omega \in \mathcal{C}^{0}$ вид отображения (1) сохранится, изменятся лишь структура и особенности инволюции $f$. Впервые подход, приводящий к отображениям вида (1), использовался при построении стохастической теории светособирания в физических системах - сцинтилляционных детекторах [14].

Сушествует проблема вычисления функции $f$ - инволюции (3), зависяшей от второго аргумента как от параметра, $f\left(\varphi_{1}, \varphi_{2}\right) \equiv f_{\varphi_{2}}\left(\varphi_{1}\right)$, по заданной форме границы $\partial \Omega$. В предлагаемой здесь постановке она определяет прямую задачу для бильярда. Сравнительно просто эта задача решается (в явном виде) только в некоторых случаях, например для интегрируемых бильярдов эллипса и окружности - кривых второго порядка с выделенными проективными свойствами (см., например, [15]). Явный вид уравнений движения и (или) отображений доступен для бильярдов в полигонах (рациональных многоугольниках) с выпрямляемыми по Шварцу траекториями, а также для бильярдов с плиссируемой гранищей, таких как стадион Бунимовича или “гусеница" (подробнее см., например, [9]). В данной работе получено общее выражение для инволюции бильярда с произвольной границей (не обязательно гладкой или строго выпуклой).

Прямая задача, безусловно, важна, так как к отображению (1) с известной инволюцией можно эффективно применить аналитические и качественные методы исследования динамических систем.

К исследованию бильярдов можно подойти и с другой стороны. Допустим, известна инволюция какой-то бильярдной системы. Например, в качестве $f$ можно использовать формальный степенной ряд с коэффициентами, обеспечиваюшими выполнение основного условия $f \circ f=\mathrm{id}$. Отметим, что и в этом случае не всякая инволюция соответствует математическому бильярду. Тогда возникает обратная задача - восстановить гранищу бильярда по заданной инволюции. Решения прямой и обратной задач должны быть взаимно согласованы между собой: $\left.\left.\vec{r}(\varphi)\right|_{\partial \Omega} \Rightarrow f\left(\varphi_{1}, \varphi_{2}\right) \Rightarrow \vec{r}(\varphi)\right|_{\partial \Omega}$.

Важным шагом на пути установления геометро-динамических закономерностей бильярда служат вывод уравнений для бильярдной инволюции и принципиальное решение прямой и обратной задач. Данная работа связана именно с этой частью развиваемой теории. 


\section{2. ИНВОЛЮЦИЯ ДВУМЕРНОГО БИЛЬЯРДА}

Любой бильярд на плоской области, ограниченной замкнутой самонепересекающейся кривой $\partial \Omega$ (границей), однозначно определяется заданием его инволюции. Геометрически инволюция связывает координаты падающего и отраженного лучей (точнее, координаты точек их пересечения с границей бильярда) относительно фиксированной точки (отражения) на границе $\partial \Omega$. Существуют различные (эквивалентные) представления бильярдной инволюции. В частности, конкретный вид инволюции, как и определяющих ее уравнений, зависит от выбора системы отсчета, способа параметризации $\partial \Omega$ и характера самих геометрических координат (в данном случае на евклидовой плоскости). В многомерном случае или для бильярда на поверхностях с кривизной аналогичную роль играет выбор атласа карт для многообразий $\Omega$ и $\partial \Omega$, на которых происходит бильярдное движение. Далее рассматривается только плоский (двумерный) бильярд, хотя его проективные особенности распространяются и на обший случай.

Исходным для всех представлений инволюции является алгебраическое соотношение, выражающее основное геометрическое условие для бильярда, - угол падения равен углу отражения. Используя скалярное $(\overrightarrow{,}, \vec{\cdot})$ и векторное $[\vec{\cdot}, \vec{\cdot}]$ произведения в евклидовом пространстве $\mathbb{R}^{3} \supset \mathbb{R}^{2} \supset \partial \Omega$, для двумерного бильярда это условие удобно представить в виде

$$
\frac{\left(\vec{n}_{2}, \vec{r}_{3}-\vec{r}_{2}\right)}{\left|\left[\vec{n}_{2}, \vec{r}_{3}-\vec{r}_{2}\right]\right|}=-\frac{\left(\vec{n}_{2}, \vec{r}_{2}-\vec{r}_{1}\right)}{\left|\left[\vec{n}_{2}, \vec{r}_{2}-\vec{r}_{1}\right]\right|}
$$

где векторы $\vec{r}_{i}=\vec{r}\left(\varphi_{i}\right), i=1,2,3$, определяют три последовательных точки бильярдной траектории на границе $\partial \Omega$, параметризованной периодическим (период $2 \pi$ выбирается для удобства) гомеоморфизмом $[0,2 \pi] \stackrel{\varphi}{\longrightarrow} \partial \Omega \subset \mathbb{R}^{2} ; \vec{r}(\varphi+2 \pi)=\vec{r}(\varphi) ; \vec{n}=\left.\vec{n}(\vec{r})\right|_{\partial \Omega}$ - поле внешних (единичных, $\vec{n}^{2}=1$ ) нормалей к $\partial \Omega$, причем $\vec{n}_{2}=\vec{n}\left(\varphi_{2}\right)$ - нормаль в фиксированной точке отражения $\vec{r}_{2}=\vec{r}\left(\varphi_{2}\right) ;|\vec{\cdot}|=\sqrt{(\vec{\cdot}, \vec{\cdot})}$ обозначает длину вектора. В декартовых координатах $\vec{r}=(x, y), \vec{n}=\left(n_{x}, n_{y}\right)$ вместо обшего соотношения (4) получим уравнение для инволюции бильярда $\varphi_{3}=f\left(\varphi_{1}, \varphi_{2}\right)$, записанное в форме неявной функции:

$$
\begin{aligned}
& \frac{x\left(f\left(\varphi_{1}, \varphi_{2}\right)\right)-x\left(\varphi_{2}\right)}{y\left(f\left(\varphi_{1}, \varphi_{2}\right)\right)-y\left(\varphi_{2}\right)}= \\
& \quad=-\frac{\left(n_{x}^{2}\left(\varphi_{2}\right)-n_{y}^{2}\left(\varphi_{2}\right)\right)\left(x\left(\varphi_{2}\right)-x\left(\varphi_{1}\right)\right)+2 n_{x}\left(\varphi_{2}\right) n_{y}\left(\varphi_{2}\right)\left(y\left(\varphi_{2}\right)-y\left(\varphi_{1}\right)\right)}{\left(n_{x}^{2}\left(\varphi_{2}\right)-n_{y}^{2}\left(\varphi_{2}\right)\right)\left(y\left(\varphi_{2}\right)-y\left(\varphi_{1}\right)\right)-2 n_{x}\left(\varphi_{1}\right) n_{y}\left(\varphi_{2}\right)\left(x\left(\varphi_{2}\right)-x\left(\varphi_{1}\right)\right)}
\end{aligned}
$$

причем $\vec{n}(\varphi)=\left(-y^{\prime}(\varphi), x^{\prime}(\varphi)\right) / \sqrt{{x^{\prime}}^{2}+y^{\prime 2}}, \vec{r}^{\prime} \equiv d \vec{r} / d \varphi$. Заменой переменных в (5) можно получить уравнение инволюции в произвольных координатах. Например, для границы $\partial \Omega$, заданной в виде графика функции $\partial \Omega=\Gamma=\{(x, y) \mid y=g(x)\}$, вместо (5) имеем в $x$-параметризации

$$
\frac{f\left(x_{1}, x_{2}\right)-x_{2}}{g\left(f\left(x_{1}, x_{2}\right)\right)-g\left(x_{2}\right)}=-\frac{\left(g^{\prime 2}\left(x_{2}\right)-1\right)\left(x_{2}-x_{1}\right)-2 g^{\prime}\left(x_{2}\right)\left(g\left(x_{2}\right)-g\left(x_{1}\right)\right)}{\left(g^{\prime}\left(x_{2}\right)-1\right)\left(g\left(x_{2}\right)-g\left(x_{1}\right)\right)+2 g^{\prime}\left(x_{2}\right)\left(x_{2}-x_{1}\right)},
$$

так как $\vec{n}=\vec{n}(x)=\left(-g^{\prime}(x), 1\right) / \sqrt{1+g^{\prime 2}}, g^{\prime} \equiv d g / d x$. 
Принципиальным свойством уравнений (4)-(6), как и любых других, получаемых из них, является их инвариантность относительно замены

$$
\varphi_{1} \rightarrow \varphi_{3}=f\left(\varphi_{1}, \varphi_{2}\right), \quad \varphi_{3} \rightarrow f\left[f\left(\varphi_{1}, \varphi_{2}\right), \varphi_{2}\right] \equiv \varphi_{1}
$$

что и означает инволютивность $f \circ f=\mathrm{id}$ (или более общо, обратимость) бильярда как динамической системы.

Не менее важен проективный характер бильярдной инволюции. Введем вместо $\left(\varphi_{1}, \varphi_{2}\right)$ и $\left(\varphi_{2}, \varphi_{3}=f\left(\varphi_{1}, \varphi_{2}\right) \equiv f\right)$ новые координаты

$$
\left(p=\frac{x\left(\varphi_{2}\right)-x\left(\varphi_{1}\right)}{y\left(\varphi_{2}\right)-y\left(\varphi_{1}\right)}, q=\varphi_{2}\right), \quad\left(\bar{p}=\frac{x(f)-x\left(\varphi_{2}\right)}{y(f)-y\left(\varphi_{2}\right)}, \bar{q}=f\right),
$$

в которых соотношение (5) примет вид однопараметрической дробно-рациональной инволюции

$$
\begin{gathered}
\bar{p}=R_{q}(p)=\frac{\alpha(q) p+\beta(q)}{\beta(q) p-\alpha(q)}, \quad R \circ R=\mathrm{id}\left(R_{q}\left(R_{q}(p)\right)=p\right), \\
\alpha=\alpha(q)=n_{x}^{2}(q)-n_{y}^{2}(q), \quad \beta=\beta(q)=2 n_{x}(q) n_{y}(q),
\end{gathered}
$$

так что $\alpha^{2}+\beta^{2}=1$. Как известно (см., например, [15]), дробно-рациональными функциями определяются именно проективные преобразования в неоднородных проективных, например евклидовых, координатах. Согласно (9) бильярд - локально проективное обратимое (инволюция) преобразование относительно выделенной точки отражения (точки $q=$ const в (9)). Этот же факт можно установить и непосредственно геометрически. Для сложного отношения (см. [15]) четырех лучей одного и того же пучка на евклидовой плоскости в случае бильярда для падающих $a, b, c, d$ и отраженных $a^{\prime}, b^{\prime}, c^{\prime}, d^{\prime}$ лучей имеем

$$
(a b c d)=\frac{\sin (a c)}{\sin (c b)} \frac{\sin (a b)}{\sin (b d)}=\left(a^{\prime} b^{\prime} c^{\prime} d^{\prime}\right),
$$

следовательно, сложное отношение сохраняется, и, значит, бильярд является проективным преобразованием. Взяв в качестве лучей $a, b, c, d$ в выражении (10) следующие лучи: падаюший $(i)$, нормальный $(n)$ и касательньй $(\tau)$ к границе, а также отраженньй $(f)$, получим, что

$$
(i n \tau f)=-1=(f n \tau i),
$$

следовательно, бильярд соответствует специальному случаю проективного преобразования - гармоническому преобразованию. При неподвижных (в точке отражения) нормали $n$ и касательной $\tau$ это преобразование, естественно, является инволюцией

$$
f=G(i ; n \tau), \quad G \circ G=\mathrm{id} .
$$

Отметим, что непрерывность и монотонность - отличительные черты всякого проективного преобразования. Непосредственно из (9) следует, что бильярду соответствует строго убывающая инволюция, причем аналитическая (в области своей непрерывности) по первому аргументу, $\partial R / \partial p=-(\beta p-\alpha)^{2}<0, R_{q}(p) \in C^{\infty}$. Дифференциальные свойства инволюции, а значит, и бильярда в целом зависят от регулярности коэффициентов $\alpha$ и $\beta$ в (9) и, следовательно, от гладкости поля нормалей $\vec{n}$ на границе $\partial \Omega$. 
Выделим проективную структуру произвольной бильярдной инволюции. Для этого рассмотрим переход к “проективным" координатам (8) как замену переменных (7), зависящую от параметра:

$$
(\varphi, \psi) \rightarrow\left(z_{\psi}(\varphi), \psi\right), \quad z_{\psi}(\varphi)=\frac{x(\varphi)-x(\psi)}{y(\varphi)-x(\psi)}
$$

Взаимно однозначный характер параметризации $\partial \Omega, \vec{r}=\vec{r}(\varphi)$, обеспечивает (локально) сушествование и единственность обратного к (13) преобразования

$$
z^{-1}=z^{-1}(\xi, \psi)=z_{\psi}^{-1}(\xi), \quad z^{-1} \circ z=z \circ z^{-1}=\mathrm{id}
$$

Укажем на кусочно-монотонный характер прямого $z$ и обратного $z^{-1}$ преобразований.

Используя (13), (14), инволюцию $f\left(\varphi_{1}, \varphi_{2}\right)$, входящую в уравнение $(5)$, можно представить в виде обшего соотношения

$$
z \circ f=R \circ z \Leftrightarrow f=z^{-1} \circ R \circ z
$$

где

$$
\begin{gathered}
f=f\left(\varphi_{1}, \varphi_{2}\right), \quad z=z_{\varphi_{2}}\left(\varphi_{1}\right), \quad z^{-1}=z_{\varphi_{2}}^{-1}(\xi), \\
R=R_{\varphi_{2}}(\chi)=\frac{\alpha\left(\varphi_{2}\right) \chi-\beta\left(\varphi_{2}\right)}{\beta\left(\varphi_{2}\right) \chi-\alpha\left(\varphi_{2}\right)}
\end{gathered}
$$

$\alpha$ и $\beta$ - величины, определенные в (9).

Например, в полярных координатах с границей, заданной уравнением $r=r(\varphi)$, соотношения (15) примут вид (здесь $\left.r^{\prime}=d r / d \varphi\right)$

$$
\begin{gathered}
f\left(\varphi_{1}, \varphi_{2}\right)=z_{\varphi_{2}}^{-1}\left(R_{\varphi_{2}}\left(z_{\varphi_{2}}\left(\varphi_{1}\right)\right)\right), \quad z_{\varphi_{2}}\left(\varphi_{1}\right)=\frac{r\left(\varphi_{1}\right) \cos \varphi_{1}-r\left(\varphi_{2}\right) \cos \varphi_{2}}{r\left(\varphi_{1}\right) \sin \varphi_{1}-r\left(\varphi_{2}\right) \sin \varphi_{2}} \\
\alpha(\varphi)=\frac{\left(r^{2}-r^{\prime 2}\right) \cos 2 \varphi+2 r r^{\prime} \sin 2 \varphi}{r^{2}+r^{\prime 2}}, \quad \beta(\varphi)=\frac{\left(r^{2}-r^{\prime 2}\right) \sin 2 \varphi-2 r r^{\prime} \cos 2 \varphi}{r^{2}+r^{\prime 2}} .
\end{gathered}
$$

Уравнения (15) занимают центральное место в последующем рассмотрении. Они явно устанавливают проективную структуру инволюции произвольного бильярда, сводящейся в подходящих координатах после замены $z$ к дробно-рациональной функции. Уравнение $f=z^{-1} \circ R \circ z$ по сути дает формальное решение прямой задачи определения инволюции бильярда по его заданной гранище $\partial \Omega$. Для получения $f$ остается лишь обратить известную функцию $z_{\varphi_{2}}\left(\varphi_{1}\right)$, что, правда, не всегда удается с делать в элементарном виде. Другое, эквивалентное, соотношение $z \circ f=R \circ z$ можно рассматривать как функциональное уравнение относительно неизвестной функции $z$ при заданной инволюции бильярда $f$. Согласно (13) само преобразование $z_{\varphi_{2}}\left(\varphi_{1}\right)$ связано с уравнением границы $\vec{r}=\vec{r}(\varphi)$. Таким образом, решение обоих этих уравнений, т.е. результируюшее восстановление границы $\partial \Omega$, определяет обратную задачу для бильярда. 


\section{3. ПРИМЕРЫ БИЛЬЯР ДНЫХ ИНВОЛЮЦИЙ (ПРЯМАЯ ЗАДАЧА)}

В качестве примеров аналитического решения прямой задачи получим бильярдные инволюции для известных кривых. Начнем с интегрируемого бильярда в эллипсе, заданном в полярных координатах (центр является началом координат) уравнениями $x=$ $a \cos \varphi, y=b \sin \varphi$. Подставляя их в выражение (5), получим эллиптическую инволюцию

$$
\begin{aligned}
& f\left(\varphi_{1}, \varphi_{2}\right)=-\varphi_{2}- \\
& \quad-2 \operatorname{arctg}\left\{\frac{\left[\left(a^{2}+b^{2}\right) \cos 2 \varphi_{2}-\left(a^{2}-b^{2}\right)\right] \operatorname{tg} \frac{\varphi_{1}+\varphi_{2}}{2}-2 b^{2} \sin 2 \varphi_{2}}{\left(a^{2}+b^{2}\right) \cos 2 \varphi_{2}-\left(a^{2}-b^{2}\right)+2 a^{2} \sin 2 \varphi_{2} \operatorname{tg} \frac{\varphi_{1}+\varphi_{2}}{2}}\right\} \quad(\bmod 2 \pi),
\end{aligned}
$$

которая в случае $a=b$ переходит в самую простую инволюцию окружности

$$
f\left(\varphi_{1}, \varphi_{2}\right)=2 \varphi_{2}-\varphi_{1} \quad(\bmod 2 \pi)
$$

сохраняюшую такой же вид и в натуральной параметризации $\partial \Omega$. Инволюция эллипса легко приводится к каноническому виду $f\left(\varphi_{1}, \varphi_{2}\right)=\left(z_{\varphi_{2}}^{-1} \circ R_{\varphi_{2}} \circ z_{\varphi_{2}}\right)\left(\varphi_{1}\right)$, где $z_{\varphi_{2}}\left(\varphi_{1}\right)=$ $\left(\varphi_{1}+\varphi_{2}\right) / 2$.

Следуюшими по сложности среди замкнутых алгебраических кривых являются кривые четвертого порядка. Например, для улиток Паскаля $r(\varphi)=a \cos \varphi+b, 0<a \leqslant b$ (случай $a=b$ соответствует кардиоиде); функции $z$ и $R$, определяемые величинами $\alpha=\alpha(\varphi)$ и $\beta=\beta(\varphi)$, имеют вид

$$
\begin{gathered}
z\left(\varphi_{1}, \varphi_{2}\right)=\frac{\left(a \cos \varphi_{1}+b\right) \cos \varphi_{1}-\left(a \cos \varphi_{2}+b\right) \cos \varphi_{2}}{\left(a \cos \varphi_{1}+b\right) \sin \varphi_{1}-\left(a \cos \varphi_{2}+b\right) \sin \varphi_{2}}, \\
\alpha=\frac{a^{2} \cos 4 \varphi+2 a b \cos 3 \varphi+b^{2} \cos 2 \varphi}{a^{2}+b^{2}+2 a b \cos \varphi}, \quad \beta=\frac{a^{2} \sin 4 \varphi+2 a b \sin 3 \varphi+b^{2} \sin 2 \varphi}{a^{2}+b^{2}+2 a b \cos \varphi} .
\end{gathered}
$$

Для этих кривых и таких, как синусоидальные спирали четного порядка (в том числе лемниската Бернулли), обычный овал, овал Кассини, конхоида Никодима и др., при вычислении инволюции (обрашении $z \rightarrow z^{-1}$ зависимости) возникает необходимость решить алгебраическое уравнение четвертой степени, соответствующие громоздкиевыражения мы приводить не будем. Не представляет труда выписать соотношения, определяюшие бильярдную инволюцию в других алгебраических (старше четвертого порядка) или трансцендентных кривых. Важно отметить возникаюшую при этом многолистность решения. Среди допустимых ветвей вешественной инволюции для бильярда должны отбираться те (при определенных значениях аргументов), которые удовлетворяют принципу наименьшего действия, т.е. $\left|\vec{r}\left(f\left(\varphi_{1}, \varphi_{2}\right)\right)-\vec{r}\left(\varphi_{2}\right)\right|=\min$ среди всех возможных ветвей инволюции $f$. Только для кривых второго порядка существует единственное (на каждой из двух сторон ориентированной евклидовой плоскости) решение $z^{-1}$ для обратной функции. Указанная выше многозначность ветвей инволюции для произвольных кривых $\partial \Omega$ тесно связана со стохастичностью (необратимостью) возникающего сложного динамического поведения в неинтегрируемых бильярдах общего положения. 


\section{4. ОБРАТНАЯ ЗАДАЧА}

Обратная задача, как и прямая, связана с проективным представлением бильярдной инволюции соотношением (15). По постановке задачи, если бильярду соответствует инволюция $f\left(\varphi_{1}, \varphi_{2}\right)=f_{\varphi_{2}}\left(\varphi_{1}\right)$, то его граница $\partial \Omega$, заданная на декартовой плоскости выражением $\vec{r}=\vec{r}(\varphi)$, удовлетворяет уравнению

$$
z_{\varphi_{2}}\left[f_{\varphi_{2}}\left(\varphi_{1}\right)\right]=R_{\varphi_{2}}^{B}\left[z_{\varphi_{2}}\left(\varphi_{1}\right)\right]
$$

с симметризованной величиной

$$
z_{\varphi_{2}}\left(\varphi_{1}\right)=z_{\varphi_{1}}\left(\varphi_{2}\right)=\frac{\left(\vec{r}\left(\varphi_{1}\right), \vec{e}_{x}\right)-\left(\vec{r}\left(\varphi_{2}\right), \vec{e}_{x}\right)}{\left(\vec{r}\left(\varphi_{1}\right), \vec{e}_{y}\right)-\left(\vec{r}\left(\varphi_{2}\right), \vec{e}_{y}\right)}
$$

и дробно-рациональной инволюцией бильярда

$$
R_{\varphi}^{B}(z)=\frac{a(\varphi) z+b(\varphi)}{b(\varphi) z-a(\varphi)}, \quad a(\varphi)=n_{x}^{2}(\varphi)-n_{y}^{2}(\varphi), \quad b(\varphi)=2 n_{x}(\varphi) n_{y}(\varphi) .
$$

Задание инволюции определяет не только форму гранишы, но и конкретный выбор координатного представления, а следовательно, способ параметризации и характер расположения контура $\partial \Omega$ на плоскости относительно выделенной системы отсчета. Система координат, в которой граница задается некоторым аналитическим уравнением, также непосредственно связана с некоторым представлением инволюции (связана с характером и видом зависимости инволюции от соответствующих координат).

Рассмотрим локальный случай, при котором точка отражения фиксирована, т.е. в уравнении $(22) \varphi_{2}=\varphi_{0}$. Пусть граница задана в виде графика функции $y=y(x)$ и величины $\vec{r}_{0}=\vec{r}\left(\varphi_{0}\right)=\left(x_{0}, y_{0}\right), \vec{n}_{0}=\vec{n}\left(\varphi_{0}\right)=\left(-y_{0}^{\prime}, 1\right), y_{0}=y\left(x_{0}\right)$ и $y_{0}^{\prime}=y^{\prime}\left(x_{0}\right)$ определяют указанную точку. Упростим начальное условие выбором специальной системы отсчета, в которой будем искать уравнение границы. Перенос начала отсчета в выделенную точку $\vec{r}_{0}$ на $\partial \Omega$ в новых координатах дает $\vec{r}_{0}=0 ;$ поворотом координатных осей добиваемся, чтобы $y_{0}^{\prime}=0\left(\vec{n}=\vec{e}_{x}\right)$ или $y_{0}^{\prime}=1\left(\vec{n}=-\vec{e}_{y}\right)$, причем базисные реперы $\{\vec{n}, \vec{\tau}\}$ и $\left\{\vec{e}_{x}, \vec{e}_{y}\right\}$ задают одну и ту же ориентацию $\partial \Omega$. Для соответствующих одномерных инволюций $f_{1}$ и $f_{2}$ (в обоих случаях $\left.x_{0}=0\right)$ из выражения $(22)$ получим

$$
\frac{y\left(f_{1}(x)\right)}{f_{1}(x)}=-\frac{y(x)}{x} \quad\left(\vec{n}=\vec{e}_{x}\right), \quad \frac{y\left(f_{2}(x)\right)}{f_{2}(x)}=\frac{x}{y(x)} \quad\left(\vec{n}=-\vec{e}_{y}\right) .
$$

В полярных координатах $x=r(\phi) \cos \phi$ и $y=r(\phi) \sin \phi$ имеем $f(x)=r(f(\phi)) \cos f(\phi)$ и $y(f(x))=r(f(\phi)) \sin f(\phi)$, а уравнения примут вид

$$
f_{1}(\phi)=-\phi, \quad f_{2}(\phi)=\operatorname{arctg}(\operatorname{ctg} \phi) \equiv \frac{\pi}{2}-\phi,
$$

где сдвиг на $\pi / 2$ соответствует повороту (против часовой стрелки) от $\vec{e}_{1 x}=\vec{n}$ до $\vec{e}_{2 x}=\vec{\tau}$. Инволюция выглядит так же, как и в бильярде на соприкасающейся (в точке $\left.\vec{r}_{0}\right)$ окружности $\left(f_{\phi_{0}}(\phi)=2 \phi_{0}-\phi\right)$. Сама граница $\partial \Omega$ при этом может быть произвольной, так как ее зависимость $r=r(\phi)$ выпадает из выражений $(25),(26)$ (но это 
происходит только в полярных координатах). Такая неоднозначность не позволяет восстановить уравнение границы при локальном решении задачи.

В обшем случае (произвольных систем координат) соотношения (22)-(26) с фиксированным начальным условием $\left\{\vec{r}_{0}, \vec{n}_{0}\right\}$ сводятся к функциональному уравнению

$$
z(f(x))=-z(x), \quad f(f(x))=x, \quad f_{x}^{\prime}<0,
$$

частное решение которого представимо в виде

$$
z(x)=z_{a}(x)=F\left(\ln \left(-\frac{d f}{d x}\right)\right), \quad F(x)=-F(-x),
$$

где $F$ - произвольная нечетная функция. Решения уравнения (27) образуют линейное подпространство в множестве кусочно-непрерывных функций. Любая такая функция допускает линейное разбиение на четную и нечетную части $z(x)=z_{c}(x)+z_{a}(x)$, $z_{c}(x)=z_{c}(-x), z_{a}(x)=-z_{a}(-x)$. (Для функций с разрывами при построении $z_{c}(x)=$ $[z(x)+z(-x)] / 2$ и $z_{a}(x)=[z(x)-z(-x)] / 2$ следует воспользоваться их симметричным продолжением относительно неподвижной точки инволюции $f, f\left(x^{*}\right)=x^{*}$. Для убывающих инволюций такая точка всегда существует.) Антисимметричная часть произвольного решения уравнения (27) сводится к представлению (28). Оставшаяся симметричная часть $z_{c}(x)$ (разность решений $z(x)-z_{a}(x)$ снова должна быть решением) должна исчезать, так как ни одна четная функция не может быть решением (27). Действительно, в противном случае из выражений $z_{c}(-f(x))=-z_{c}(-(-x)) \equiv-z_{c}(-x)=$ $z_{c}(f(-x))$ и $z_{c}(f(-x))=-z_{c}(-x) \equiv-z_{c}(x)=z_{c}(f(x))$, справедливых при произвольных $( \pm x)$ и $( \pm f( \pm x))$, следует противоречие: с одной стороны, $f(x)=-f(-x)$, а с другой стороны, $f(x)=f(-x)$. Таким образом, выражение $(28)$ дает обшее решение линейного уравнения $(27)$.

Попробуем доопределить произвольную (вспомогательную) величину $F$ из условия согласования прямой и обратной задач. Это условие означает, что решение $z$ уравнения (22), полученное в виде (28), должно тождественно удовлетворять исходному проективному соотношению (15) $f=z^{-1} \circ R^{B} \circ z$ для инволюции $f$. Введем функцию $\zeta(x)=\ln (-d f / d x)$. Точки, в которых $\zeta_{x}^{\prime}=0$, соответствуют $f_{x^{2}}^{\prime \prime}=0$ или $n_{x}(\vec{r}) n_{y}(\vec{r})=0$ (для доказательства следует воспользоваться представлением инволюции в дробнорациональном виде). Число таких точек, в которых $n_{x}=0$ или $n_{y}=0$, для замкнутой ограниченной кривой конечно. Следовательно, локально существует обратная функция $\zeta^{-1}(x), \zeta \circ \zeta^{-1}=\zeta^{-1} \circ \zeta=\mathrm{id}$. С помошью представления $F=z \circ \zeta^{-1}$ из условия самосогласования получим уравнение

$$
F(g(x))=R^{B}(F(x)), \quad g=\zeta \circ f \circ \zeta^{-1}, \quad g \circ g=\mathrm{id}, \quad g_{x}^{\prime}<0,
$$

с вновь образованной инволюцией $g$ и прежней $R^{B}$, определенной в (24). После замены $F(x)=\operatorname{tg}[w(x)-(1 / 2) \operatorname{arctg}(b / a)]$ приходим к уравнениям

$$
z(f(x))=-z(x) \Rightarrow w(g(x))=-w(x) \Rightarrow w(f(x))=-w(x),
$$

где последний переход обусловлен симметрией $\zeta(f(x))=-\zeta(x)$, следующей из тождества $f_{f}^{\prime} f_{x}^{\prime}=1(f \circ f=\mathrm{id})$, откуда $g(f(x))=-g(x)$, а также нечетным характером 
общего решения, $w(x)=G\left(\ln \left(-g_{x}^{\prime}\right)\right)$ и $G(x)=-G(-x)$, для полученного уравнения c $w$. Повторяя проведенный вывод, но уже для величины $G$, получим следующую вспомогательную неизвестную и т.д. Оборвать эту незамкнутую цепочку и однозначно восстановить границу бильярда можно, как уже отмечалось, лишь вернувшись к исходным функциональным уравнениям (22)-(24) в нелокальной постановке задачи.

Предварительно рассмотрим более общий, чем уравнение (22), тип функциональных уравнений с дробно-рациональной функцией $R$ и инволюцией $f$ :

$$
z(f(x))=R(z(x))=\frac{a z(x)+b}{c z(x)+d}, \quad f \circ f=\mathrm{id},
$$

где $z, f$ и коэффициенты $a, b, c, d$ могут зависеть от параметра. В классе кусочногладких (непрерывно дифференцируемых) функций его решения сушествуют не всегда. Для дробно-рациональной инволюции $(a \equiv-d)$

$$
z(f(x))=R^{I}(z(x))=\frac{a z(x)+b}{c z(x)-a}, \quad R^{I} \circ R^{I}=\mathrm{id}
$$

решения появляются парами. Непосредственно из уравнения (32) следует, что если $z_{1}=z(x)$ является его решением, то и $z_{2}=z(f(x)) \neq z(x)$ будет таковым, так как при замене $x \leftrightarrow f(x)$ в исходном уравнении оно переходит в $z(x)=R^{I}(z(f(x)))$. Вырожденный случай $f(x)= \pm x$ тривиален и не рассматривается. Совместное сушествование инволютивно-сопряженных решений требует вьполнения

$$
\left(a^{2}+b c\right)^{4}=1, \quad \frac{z_{f}^{\prime}(f)}{z_{x}(x)}=-\frac{a^{2}+b c}{(c z(x)-a)^{2}} \frac{1}{f_{x}^{\prime}(x)},
$$

откуда с учетом соотношения $\operatorname{sign} z_{f}^{\prime}=\operatorname{sign} z_{x}^{\prime}\left(z_{f}^{\prime} z_{x}^{\prime}=1\right)$ для рассматриваемых здесь вешественных функций следует $\left(a^{2}+b c\right)=-\operatorname{sign} f_{x}^{\prime}$, т.е. условие "нормировки" для коэффициентов проективной инволюции $R^{I}$. Для бильярда получаем (см. (15)) $b \equiv c$, что соответствует строго убывающей инволюции

$$
R^{B}(x)=\frac{a x+b}{b x-a}, \quad a^{2}+b^{2}=-\operatorname{sign} f_{x}^{\prime}=+1 .
$$

Заменой $z(x)=\operatorname{tg}[w(x)-(1 / 2) \operatorname{arctg}(b / a)]$ уравнение $(32)$ с бильярдной инволюцией (34) приводится к виду $w(f(x))=-w(x)$ с известным из предыдушего анализа решением $w(x)=F\left(\ln \left(-f_{x}^{\prime}\right)\right)$.

После преобразований получим общее решение уравнения (22) (в последнем можно убедиться непосредственной подстановкой)

$$
z_{\varphi_{2}}\left(\varphi_{1}\right)=\frac{x\left(\varphi_{1}\right)-x\left(\varphi_{2}\right)}{y\left(\varphi_{1}\right)-y\left(\varphi_{2}\right)}=\frac{n_{x}\left(\varphi_{2}\right) \operatorname{tg}\left[F_{\varphi_{2}}\left(\ln \left|f_{\varphi_{1}}^{\prime}\left(\varphi_{1}, \varphi_{2}\right)\right|\right)\right]-n_{y}\left(\varphi_{2}\right)}{n_{y}\left(\varphi_{2}\right) \operatorname{tg}\left[F_{\varphi_{2}}\left(\ln \left|f_{\varphi_{1}}^{\prime}\left(\varphi_{1}, \varphi_{2}\right)\right|\right)\right]+n_{x}\left(\varphi_{2}\right)}
$$

с неопределенной пока величиной $F_{\varphi_{2}}\left(\varphi_{1}\right)=-F_{\varphi_{2}}\left(-\varphi_{1}\right)$, заданной функцией $f_{\varphi_{1}}^{\prime}=$ $\partial f\left(\varphi_{1}, \varphi_{2}\right) / \partial \varphi_{1}$ и неизвестными уравнениями границы $\vec{r}(\varphi)$ и поля нормалей $\vec{n}(\varphi)$ к ней.

В самом обшем случае граница $\partial \Omega$ задается параметрически, т.е. уравнениями $\vec{r}=$ $\vec{r}(t)$ и (или) $\vec{n}=\vec{n}(t)$ (роль $\varphi$ играет параметр $t$ ). Введем переменную $\xi(t)=y_{t}^{\prime} / x_{t}^{\prime}=$ 
$d y / d x$ - тангенс угла наклона касательной $\vec{\tau}(t)$ к $\partial \Omega$. Переход от переменной $t$ к $\xi(t)$ означает переход к проективно-двойственным образам, т.е. от точки с координатами $\vec{r}(t)$ к лучу с направляющей $\vec{n}(t)$. Новая параметризация границы $\partial \Omega$ в координатах направляющих (или касательных) поля нормалей не зависит от выбора координатного представления, а определяется только формой границы $\partial \Omega$. Это позволяет решить в обшем случае уравнение (35) относительно неизвестной величины $\vec{n}(t)$. Получить прямое решение этого же уравнения, но относительно координатной величины $\vec{r}(t)$, не столь просто. В новых обозначениях имеем

$$
\begin{gathered}
\xi\left(t_{1}\right)=\frac{A\left(t_{1}, t_{2}\right) \xi\left(t_{2}\right)-B\left(t_{1}, t_{2}\right)}{B\left(t_{1}, t_{2}\right) \xi\left(t_{2}\right)+A\left(t_{1}, t_{2}\right)} \Leftrightarrow \xi\left(t_{2}\right)=-\frac{A\left(t_{1}, t_{2}\right) \xi\left(t_{1}\right)+B\left(t_{1}, t_{2}\right)}{B\left(t_{1}, t_{2}\right) \xi\left(t_{1}\right)-A\left(t_{1}, t_{2}\right)} \\
A\left(t_{1}, t_{2}\right)=1+\operatorname{tg}\left[F\left(\ln \left|f_{t_{1}}^{\prime}\right|\right)\right] \operatorname{tg}\left[F\left(\ln \left|\widetilde{f}_{t_{2}}^{\prime}\right|\right)\right] \\
B\left(t_{1}, t_{2}\right)=\operatorname{tg}\left[F\left(\ln \left|f_{t_{1}}^{\prime}\right|\right)\right]-\operatorname{tg}\left[F\left(\ln \left|\tilde{f}_{t_{2}}^{\prime}\right|\right)\right]
\end{gathered}
$$

где $\tilde{f}\left(t_{1}, t_{2}\right) \equiv f\left(t_{2}, t_{1}\right)$. Отметим симметрию величин $A\left(t_{1}, t_{2}\right)=A\left(t_{2}, t_{1}\right)$ и $B\left(t_{1}, t_{2}\right)=$ $-B\left(t_{2}, t_{1}\right)$ и самих уравнений (36) при замене $t_{1} \leftrightarrow t_{2}$. Последняя играет роль инволютивной замены $t_{1} \leftrightarrow f_{t_{2}}\left(t_{1}\right)$, так как соотношение $\xi\left(f_{t_{2}}\left(t_{1}\right)\right)=\xi\left(t_{2}\right)$ прямо следует из $(36)$ с учетом определения (37) и свойства $f_{f}^{\prime} \equiv x_{f}^{\prime}=1 / f_{x}^{\prime}$ для инволюции.

Решение уравнения (36) получается в квадратурах:

$$
\xi(t)=\operatorname{tg} \int_{t_{0}}^{t} \frac{A_{t}^{\prime}\left(t, t_{0}\right) B\left(t, t_{0}\right)-A\left(t, t_{0}\right) B_{t}^{\prime}\left(t, t_{0}\right)}{A^{2}\left(t, t_{0}\right)+B^{2}\left(t, t_{0}\right)} d t,
$$

где зависимость от $t_{0}$ следует считать зависимостью от начального условия. Естественно, форма границы $\partial \Omega$ не должна зависеть от выбора начальной точки $t_{0}$ на этой кривой. Это означает, что в действительности правая часть (38) от $t_{0}$ не должна зависеть. Так как эта зависимость неявная и определяется величиной $F_{t_{0}}(t)$, которая входит в коэффициенты $A$ и $B$, то это накладывает определенные ограничения на вид $F$ и, следовательно, на вид инволюции бильярда $f$ ( $F$ и $f$ связаны условием согласования прямой и обратной задач). Дифференцируя выражение (38) по $t_{0}$, получим одно из таких условий. После алгебраических преобразований оно представимо в виде

$$
A_{t_{1} t_{2}}^{\prime \prime}\left(t_{1}, t_{2}\right)=A_{t_{2} t_{1}}^{\prime \prime}\left(t_{1}, t_{2}\right)
$$

что предполагает соответствующую регулярность инволюции $f \in \mathcal{C}^{3}$ (граница $\partial \Omega$ класса $\mathcal{C}^{2}$ с непрерывной кривизной) и коммутативность смешанных производных

$$
f_{t_{1} t_{2}}^{\prime \prime}\left(t_{1}, t_{2}\right)=f_{t_{2} t_{1}}^{\prime \prime}\left(t_{1}, t_{2}\right), \quad f \in \mathcal{C}^{3} \Leftrightarrow \partial \Omega \in \mathcal{C}^{2} .
$$

Эти требования выполняются для бильярдной инволюции, представленной в дробнорациональном виде $(9)$ для гладкой границы класса $\mathcal{C}^{2}$.

Для однозначного решения обратной задачи необходимо доопределить величину $F$, уравнение для которой получается из условия самосогласования, представленного, как и при проведенном ранее локальном анализе, в виде $f=z^{-1} \circ R^{B} \circ z$. Перед его применением необходимо получить само уравнение границы $\vec{r}(t)$, считая формально $F_{t_{2}}\left(t_{1}\right)$ известной величиной. 
В произвольной параметризации границы $\varphi=t$ и $\vec{r}=\vec{r}(t)$, обозначив полученное ранее выражение $(35)$ для $z_{t_{2}}\left(t_{1}\right)$ через $z_{t_{2}}\left(t_{1}\right)=D\left(t_{1}, t_{2}\right)$, после его дифференцирования приходим к уравнениям

$$
\begin{gathered}
x^{\prime}\left(t_{1}\right)=-R_{x}\left(t_{1}, t_{2}\right) x^{\prime}\left(t_{2}\right), \quad y^{\prime}\left(t_{1}\right)=-R_{y}\left(t_{1}, t_{2}\right) y^{\prime}\left(t_{2}\right), \\
R_{x}\left(t_{1}, t_{2}\right)=\frac{D_{t_{1}}^{\prime}\left(t_{1}, t_{2}\right)}{D_{t_{2}}^{\prime}\left(t_{1}, t_{2}\right)} \frac{1-\xi\left(t_{2}\right) D\left(t_{1}, t_{2}\right)}{1-\xi\left(t_{1}\right) D\left(t_{1}, t_{2}\right)}, \quad R_{y}\left(t_{1}, t_{2}\right)=R_{x}\left(t_{1}, t_{2}\right) \frac{\xi\left(t_{1}\right)}{\xi\left(t_{2}\right)},
\end{gathered}
$$

откуда после интегрирования получим

$$
x(t)=x_{0}+\int_{t_{0}}^{t} R_{x}\left(t, t_{0}\right) d t, \quad y^{\prime}(t)=y_{0}+\int_{t_{0}}^{t} R_{y}\left(t, t_{0}\right) d t, \quad \vec{r}_{0}=\vec{r}\left(t_{0}\right) .
$$

Представим для удобства полученные результаты в операторном виде. Введем новую величину $\Sigma\left(t_{1}, t_{2}\right)=\Sigma_{t_{2}}\left(t_{1}\right) \equiv F_{t_{2}}\left(\ln \left(-f_{t_{2}}^{\prime}\left(t_{1}, t_{2}\right)\right)\right)$. Раскрывая производные в выражении $(38)$ с учетом определений (37), получим соотношение

$$
\left[\operatorname{arctg} \xi\left(t_{1}\right)\right]_{t_{1}}^{\prime}=\Sigma_{t_{1}}^{\prime}\left(t_{1}, t_{2}\right)-\Sigma_{t_{1}}^{\prime}\left(t_{2}, t_{1}\right)
$$

В результате интегрирования (44) проективная величина $\xi(t)$ (поле касательных или нормалей к гранище) может быть представлена в виде

$$
\xi(t)=\frac{\tau_{x}(t)}{\tau_{y}(t)}=-\frac{n_{y}(t)}{n_{x}(t)} \stackrel{\text { def }}{\equiv} \widehat{\Xi}\left[\Sigma\left(t, t_{0}\right)\right] \equiv-\operatorname{tg}\left\{\Sigma\left(t, t_{0}\right)-\Sigma\left(t_{0}, t\right)-\operatorname{arctg} \xi\left(t_{0}\right)\right\} .
$$

Из условия симметрии $z\left(t_{1}, t_{2}\right)=z\left(t_{2}, t_{1}\right)$ для выражения $(35)$ получим

$$
\begin{aligned}
\Sigma_{t_{2}}\left(t_{1}\right)-\Sigma_{t_{1}}\left(t_{2}\right) & =-\operatorname{arctg} \frac{\xi\left(t_{1}\right)-\xi\left(t_{2}\right)}{1+\xi\left(t_{1}\right) \xi\left(t_{2}\right)} \equiv \\
& \equiv \operatorname{arctg}\left[\frac{n_{x}\left(t_{1}\right) n_{y}\left(t_{2}\right)-n_{x}\left(t_{2}\right) n_{y}\left(t_{1}\right)}{n_{x}\left(t_{1}\right) n_{x}\left(t_{2}\right)+n_{y}\left(t_{2}\right) n_{y}\left(t_{1}\right)}\right] .
\end{aligned}
$$

Прямой проверкой можно убедиться в эквивалентности соотношений (45) и (46).

Учитывая, что ядра $R_{x}$ и $R_{y}$, входяшие в (43), определяются инволюцией $f$ и величиной $F$ или, что то же самое, величиной $\Sigma$ согласно выражениям $(35),(37)$ и $(38)$, в операторной записи можно представить и уравнение границы $\vec{r}=\vec{r}(t) \equiv \vec{r}\left(\Sigma_{t_{0}}(t)\right)$ :

$$
x(t)=\widehat{X}\left[\Sigma_{t_{0}}(t)\right], \quad y(t)=\widehat{Y}\left[\Sigma_{t_{0}}(t)\right] .
$$

Условие для определения $\Sigma\left(t_{1}, t_{2}\right)$, а значит, и $F\left(t_{1}, t_{2}\right)$ выглядит как соотношение $z\left(t_{1}, t_{2}\right)=\operatorname{tg} \Sigma\left(t_{1}, t_{2}\right)$, что эквивалентно исходным соотношениям $z \circ f=R^{B} \circ z$ или $f=$ $z^{-1} \circ R^{B} \circ z$. В операторных обозначениях оно примет вид функционального уравнения относительно величины $\Sigma_{t_{0}}(t)$

$$
\frac{\widehat{X}\left[\Sigma_{t_{0}}(t)\right]-X_{0}}{\widehat{Y}\left[\Sigma_{t_{0}}(t)\right]-Y_{0}}=\operatorname{tg} \Sigma_{t_{0}}(t)
$$

с условиями $X_{0}=\left.\widehat{X}\left[\Sigma_{t_{0}}(t)\right]\right|_{t=t_{0}}$ и $Y_{0}=\left.\widehat{Y}\left[\Sigma_{t_{0}}(t)\right]\right|_{t=t_{0}}$. 
Фактически в уравнение $(48)$ кроме $\Sigma_{t_{0}}(t)$ входит сопряженная величина $\Sigma_{t}\left(t_{0}\right)$ (ее появление можно рассматривать как результат действия оператора перестановки аргументов $\left.t \leftrightarrow t_{0}\right)$. Поэтому для их определения одного уравнения (48) со скрытыми первыми производными (ими согласно соотношению (46) определяется связь между $\Sigma_{t_{0}}(t)$ и $\left.\Sigma_{t}\left(t_{0}\right)\right)$ недостаточно. Система уравнений станет замкнутой, если рассмотреть вторые производные, как это и должно быть при полном описании собственной геометрии кривых на плоскости. Перепишем уравнение (46) в операторном виде

$$
\Sigma_{t_{0}}(t)-\Sigma_{t}\left(t_{0}\right)=\operatorname{arctg}\left[\frac{\widehat{X}_{t}^{\prime} Y_{0}^{\prime}-X_{0}^{\prime} \widehat{Y}_{t}^{\prime}}{\widehat{X}_{t}^{\prime} X_{0}^{\prime}+Y_{0}^{\prime} \widehat{Y}_{t}^{\prime}}\right],
$$

где

$$
\widehat{X}_{t}^{\prime}=\frac{\partial}{\partial t} \widehat{X}\left[\Sigma_{t_{0}}(t)\right] \equiv x^{\prime}(t), \quad \widehat{Y}_{t}^{\prime}=\frac{\partial}{\partial t} \widehat{Y}\left[\Sigma_{t_{0}}(t)\right] \equiv y^{\prime}(t),
$$

а $X_{0}^{\prime}=\left.\widehat{X}_{t}^{\prime}\right|_{t=t_{0}}$ и $Y_{0}^{\prime}=\left.\widehat{Y}_{t}^{\prime}\right|_{t=t_{0}}$ вместе с $X_{0}$ и $Y_{0}$ составляют "начальное условие" для произвольно выбранной точки на кривой $\partial \Omega$.

Рассмотрим производную проективной координаты $\xi_{t}^{\prime}(t)$. Используя ее и саму величину $\xi(t)$, можно составить метрический инвариант искомой кривой - кривизну, определяющую форму границы. Собственным инвариантом кривой будет кривизна в натуральной параметризации $\widehat{K}(l)$ или комбинация $\widehat{K}(t) d l / d t\left(d l / d t=\sqrt{x^{\prime 2}(t)+y^{\prime 2}(t)}\right)$ в произвольной параметризации. Ориентированная (обход против часовой стрелки) кривизна $\widehat{K}$ выражается в виде

$$
\widehat{K} \frac{d l}{d t}=\frac{x_{t}^{\prime \prime}(t) y_{t}^{\prime}(t)-x_{t}^{\prime}(t) y_{t}^{\prime \prime}(t)}{x_{t}^{\prime 2}(t)+y_{t}^{\prime 2}(t)} \equiv \frac{\xi_{t}^{\prime}(t)}{1+\xi^{2}(t)} .
$$

Отметим эквивалентность уравнений (49) и (50) (второе получается в результате дифференцирования первого). Переписав последнее выражение в операторном виде с учетом очевидного соотношения $\xi^{\prime} /\left(1+\xi^{2}\right)=(\operatorname{arctg} \xi)^{\prime}$ и используя выражения (44) и (48), получим в предыдуших обозначениях недостаюшее уравнение, замыкаюшее систему для определения $\Sigma_{t_{0}}(t)$, а значит, и $F$ :

$$
\begin{array}{r}
\frac{\widehat{X}_{t^{2}}^{\prime \prime}\left[\Sigma_{t_{0}}(t)\right] \widehat{Y}_{t}^{\prime}\left[\Sigma_{t_{0}}(t)\right]-\widehat{X}_{t}^{\prime}\left[\Sigma_{t_{0}}(t)\right] \widehat{Y}_{t^{2}}^{\prime \prime}\left[\Sigma_{t_{0}}(t)\right]}{\widehat{X}_{t}^{\prime 2}\left[\Sigma_{t_{0}}(t)\right]+\widehat{Y}_{t}^{\prime 2}\left[\Sigma_{t_{0}}(t)\right]}=\Sigma_{t}^{\prime}\left(t, t_{0}\right)-\Sigma_{t}^{\prime}\left(t_{0}, t\right) \equiv \\
\equiv \frac{\widehat{X}_{t}^{\prime}\left[\Sigma_{t_{0}}(t)\right]\left(\widehat{Y}\left[\Sigma_{t_{0}}(t)\right]-\widehat{Y}_{0}\right)-\widehat{Y}_{t}^{\prime}\left[\Sigma_{t_{0}}(t)\right]\left(\widehat{X}\left[\Sigma_{t_{0}}(t)\right]-\widehat{X}_{0}\right)}{\left(\widehat{X}\left[\Sigma_{t_{0}}(t)\right]-\widehat{X}_{0}\right)^{2}+\left(\widehat{Y}\left[\Sigma_{t_{0}}(t)\right]-\widehat{Y}_{0}\right)^{2}}- \\
-\frac{\widehat{X}_{t}^{\prime}\left[\Sigma_{t}\left(t_{0}\right)\right]\left(\widehat{Y}\left[\Sigma_{t}\left(t_{0}\right)\right]-\widehat{Y}_{0}\right)-\widehat{Y}_{t}^{\prime}\left[\Sigma_{t}\left(t_{0}\right)\right]\left(\widehat{X}\left[\Sigma_{t}\left(t_{0}\right)\right]-\widehat{X}_{0}\right)}{\left(\widehat{X}\left[\Sigma_{t}\left(t_{0}\right)\right]-\widehat{X}_{0}\right)^{2}+\left(\widehat{Y}\left[\Sigma_{t}\left(t_{0}\right)\right]-\widehat{Y}_{0}\right)^{2}}
\end{array}
$$

Это будет дифференциальное уравнение второго порядка относительно неизвестной функции $\Sigma_{t_{0}}(t)$. Естественным граничным условием для него, как и для соответствующего интегрального уравнения (48), служит требование $\left.\Sigma_{t_{2}}\left(t_{1}\right)\right|_{t_{1}=t_{2}} \equiv 0$ на диагонали $t_{1}=t_{2} \quad\left(t_{2} \equiv t_{0}\right.$ - здесь уже не фиксированное значение параметра, а второй аргумент инволюции). 
Решение системы уравнений (48), (51) (первое из них выступает один раз самостоятельно, а другой раз входит во второе уравнение с кривизной) существует для кусочногладкой инволюции $f \in \mathcal{C}^{3}$. Напомним, что произвольная инволюция является строго кусочно-монотонной функцией по инволютивной переменной с отличной от нуля производной в области своей непрерывности. Согласно теореме о неявной функции уравнение (48) позволяет локально выразить $\Sigma_{t}\left(t_{0}\right)$ через $\Sigma_{t_{0}}(t)$ при всех $t \neq t_{0}$. На диагонали $t=t_{0}$ особенностей тоже не возникает, на ней инволюция одинакова для всех бильярдов и $f\left(t_{0}, t_{0}\right)=t_{0}$. После соответствуюшей подстановки в уравнение (51) его решение для величины $\Sigma_{t_{0}}(t)$ гарантируется теоремами сушествования и единственности для обыкновенных дифференциальных уравнений. Появление уравнения (51), непосредственно связанного с кривизной бильярда, не случайно и имеет глубокие геометрические причины. Как уже отмечалось, существуют различные координатные представления инволюции бильярда и его границы. Очевидно, что уравнение для восстановления гранищы по заданной инволюции бильярда должно иметь геометрически ковариантный характер и не зависеть от выбора координат. Только в этом случае можно гарантировать единственность решения обратной задачи для бильярда. Поэтому таким уравнением является уравнение для кривизны, выраженной через инволюцию бильярда, т.е. именно уравнение (51). Кривизна границы бильярда является его метрическим инвариантом и зависит только от формы границы, но не от выбора координатных переменных для этой границы или соответствующей ей инволюции бильярда. Таким образом, обратная задача имеет решение, по крайней мере, для кривых $\partial \Omega$ с непрерывным распределением кривизны. Связь инволюции бильярда с его кривизной приводит и к другим важным следствиям, в частности инвариантная мера бильярда определяется кривизной его границы.

Отметим, что из уравнения (46) и сопряженного ему (при замене $t_{1} \leftrightarrow t_{2}$ ) следует условие

$$
\Sigma_{t_{1} t_{2}}^{\prime \prime}\left(t_{1}, t_{2}\right)=\Sigma_{t_{2} t_{1}}^{\prime \prime}\left(t_{2}, t_{1}\right)
$$

с учетом коммутативности для инволюции $f_{t_{1}, t_{2}}^{\prime \prime}=f_{t_{2}, t_{1}}^{\prime \prime}$. Соотношение $(52)$ накладывает дополнительные ограничения на выбор величин $\Sigma$ или $F$. Условия $(39)$ и $(52)$ эквивалентны друг другу.

Вычисления, которые привели к системе (48), (51), в сушности, представляют собой ряд функциональных замен специального вида, которые позволяют получить аналитическое представление соответствующих геометрических образов - уравнения кривой и поля нормалей (касательных) к ней - с помощью инволюции бильярда

$$
f\left(\varphi, \varphi_{0}\right) \rightarrow z_{\varphi_{0}}\left[\Sigma_{\varphi_{0}}(\varphi)\right] \rightarrow \vec{n}\left[\Sigma_{\varphi_{0}}(\varphi)\right] \rightarrow \vec{r}\left[\Sigma_{\varphi_{0}}(\varphi)\right] \rightarrow F_{\varphi_{0}}(\varphi) \rightarrow \vec{r}(\varphi)
$$

В этой цепочке появляется вспомогательная величина $F$, связанная с произволом в решении функциональных уравнений (или выбором функциональных замен). Для ее доопределения используется согласование между инволюцией бильярда и его геометрической границей (проективная связь и связь с кривизной). 


\section{5. ЗАКЛЮЧЕНИЕ}

Перечислим основные результаты работы. С бильярдом, как с обратимой динамической системой специального вида, связано семейство инволюций, зависящих от второго аргумента как от параметра. Каждая из таких инволюций в подходящих координатах приводится к дробно-рациональной функции, что отражает проективный характер бильярдного преобразования. Связь между инволюцией бильярда и его границей определяется соответствующими выражениями в виде неявной зависимости (5) или в виде функционального уравнения специального вида (15). Решения последнего, записанного в одном из двух возможных вариантов, дают соответственно решения прямой (16), (17) (в полярных координатах) и обратной (уравнения (38) и (43) или (47) вместе с системой (48), (51)) задач бильярда. Прямая задача определяет бильярдную инволюцию по заданной границе, а обратная восстанавливает границу по известной инволюции. В обоих случаях для бильярда с достаточно гладкой границей и кривизной решения сушествуют, однозначны и согласованы друг с другом. Для простейших бильярдов инволюция вычисляется в аналитическом виде (например, кривые второго порядка). На примере решения обратной задачи демонстрируется важность связи между инволюцией бильярда и кривизной его границы.

Проективный характер и инволютивность бильярда являются теми его основными особенностями, которые сохраняются в многомерном, многосвязном и неевклидовом случаях. Наиболее важным соотношением между динамическими (инволюцией, мерой) и геометрическими характеристиками бильярда является их связь с кривизной границы бильярда (формами кривизны и связности в случае произвольных многообразий).

\section{Список литературы}

[1] Дж. Биржгоф. Динамические системы. М.: Гостехиздат, 1941.

[2] Н. С. Крылов. Работы по обоснованию статистической физики. М.: Изд-во АН СССР, 1950.

[3] Я. Г. Синай. ДАН СССР. 1963. Т. 153. № 6. С. 1261; УМН. 1970. Т. 25. № 2. С. 141.

[4] Л. А. Бунимович. Матем. сб. 1974. Т. 95. № 1. С. 49.

[5] T. Guhr, A. Muller-Groeling, H. A. Weidenmuller. Phys. Rep. 1998. V. 299. P. 1.

[6] Современные проблемы математики. Фундаментальные направления. Динамические системы-2. Т. 2. Ред. Я.Г. Синай. М.: ВИНИТИ, 1985.

[7] L. Bunimovich, G. Casati, I. Guarneri. Phys. Rev. Lett. 1996. V. 77. P. 2941.

[8] В. Ф. Лазуткин. Выпуклый бильярд и собственные функции оператора Лапласа. Л.: ЛГУ, 1981.

[9] Г. М. Заславский, Р. З. Сагдеев. Введение в нелинейную физику. От маятника до турбулентности. М.: Наука, 1988.

[10] С. Табачников. УМН. 1993. Т. 48. № 6. С. 75.

[11] V.I. Arnol'd. Reversible systems. In: Nonliner and Turbulent Processes in Physics. V. 3. Ed. R.Z. Sagdeev. Chur: Harwood, 1984. P. 1161; V. I. Arnold, M. B. Sevryuk. Oscillations and bifurcations in reversible systems. In: Nonlinear Phenomena in Plasma Physics and Hydrodynamics. M.: Mir, 1986. P. 31.

[12] M. B. Sevryuk. Reversible Systems. Lect. Notes in Math. V. 1211. Berlin: Springer, 1986.

[13] J. A. G. Roberts, G. R. W. Qwispel. Phys. Rep. 1992. V. 216. P. 1.

[14] S. V. Naidenov, V. V. Yanovskii. Funct. Materials. 2000. V. 7. № 4(2). P. 743.

[15] Н. В. Ефимов. Высшая геометрия. М.: Физматгиз, 1961. 